\title{
A Detailed survey and Enhancement in plant disease detection Using enhanced feature set
}

\author{
Ramandeep Kaur \\ Department of Computer Science and Engineering \\ IET Bhaddal Technical Campus, Ropar, Punjab,India \\ Dr.Sanjay Singla \\ Prof. \& Head CSE \\ Department of Computer Science and Engineering \\ IET Bhaddal Technical Campus, Ropar, Punjab,India
}

\begin{abstract}
This paper presents a new technique of classification of Plant Leaf Disease (Potato Late Blight) using gradient and texture features and Artificial Neural Networks. This research paper discusses and compares the two very effective techniques for plant leaf disease detection. We in this paper have made a head to head analysis of two techniques named as parallel combination method and Image contrast enhancement for the purpose of plant leaf disease detection. Both the techniques work well in their frameworks but show me some deficiencies during the dynamic changes made in context of the physical changes happening naturally. Still by a far judgment we can say that image contrast enhancement is a notch higher than its competitors. After the comparison an enhancement method is also proposed.
\end{abstract}

Keywords - component; formatting; style; styling; insert (key lwords)

\section{INTRODUCTION}

At that point fleecy $\mathrm{C}$-mean gathering is associated with piece those disease affected region which similarly join establishment for same shading qualities [1]. The dependable distinguishing proof for infection obliges constant screening of the sickness reality Eventually Tom's scrutinizing an expert which is the long run expending, drearily and Now and again not down to earth due to a little measure of bosses and A greater sum for farms. In this way, a fast late revile tainted tubers demonstrate eccentric reddish tan on purplish extents which increase under inward tissues of the tubers. At the point when a guest plunges to A site made done PHP, the server scrutinizes the PHP code and so forth systems it as expressed by its scripted headings [2]. The debased tubers when in doubt need help hard, dry Also firm Anyhow may get attacked Eventually Tom's examining sensitive rot making minute living beings Furthermore rot for field and spares.

The moment that parts from guaranteeing spots need help present, they could grow together What's more wound up A scourge or A smear. At that point that grape leaf fat division might be performed using changed self-arranging trademark map with inherited figurings for streamlining and help vector machines to arrange [3]. Infectious spots need help for the most part round or freestyle in state. Secured close by essentially cases, leaf beet spots are seen as ought to an opportunity to be only a cosmea issue, regardless they camwood need budgetary effects once nurseries on the other hand in cultivation. This is found over chestnut relinquishes.

The degree for having a place, wk(x), will be connected on the other hand of the detachment from $\mathrm{x}$ of the gathering point of convergence similarly figured on the past pasquinade. It furthermore depends on a parameter $\mathrm{m}$ that controls upon what measure of weight might be accommodated of the nearest point of convergence. Overall multifaceted nature picture redesign require much playing point However it way this nonappearance in adjacent overhaul of picture suggests it neglects to offer the area purpose of enthusiasm around a photo [4]. That calculation minimizes intra-bunch distinction comparatively too, yet all the need those same issues Likewise k-implies; those slightest will be An area least, and the impacts depend on upon those initial choice for weights.

Using A blend of Gaussians nearby those desire boost calculation might be an all the more measurably formalized framework which joins a couple of about these thoughts: deficient interest to classes. Another photo nature assessment worldview is prescribed on the backing of the Factual offers of the dim level histogram from asserting pictures with control that iterative strategy of the proposed picture redesign computation [5]. A substitute calculation 
about related to fleecy C-Means might be sensitive K-implies. They measure both those assention of as far as possible and the assurance of the division shape [6]. Taking after continually weighted likewise changed over Eventually Tom's scrutinizing a work (decided Eventually Tom's examining that system's fashioner), the initiations of these neurons need help At that point passed investigating to different neurons. This approach might be repeater until at long last; a yield neuron will be incited. This figures out which character may have been scrutinized.

Potato is a standout amongst the most exceedingly devoured crops over the whole world. The late curse is the most imperative malady for potato, which is brought about by a parasite like life form, Phytophthora infestans in muggy conditions with temperature inside a particular reach. This illness can decimate products of the whole field, bringing about significant yield misfortune, inside a couple days after the main injury shows up in the ranch. Accordingly, early recognition of this illness assault is crucial for anticipation of gigantic financial misfortunes. At that point Fuzzy C-mean grouping is connected to portion the infection influenced range which likewise incorporate foundation with same shading attributes [8]. The early Detection of sickness requires consistent observing of the ailment seriousness by a specialist which is tedious, dreary and in some cases not attainable because of a little number of specialists and a bigger number of ranches. Along these lines, a quick, dependable, and programmed strategy is required to precisely evaluate the infection seriousness.

The malady shows up as water-doused sporadic light green sores generally close tip and edges of leaves which quickly develop into huge cocoa to purplish dark necrotic spots. A white buildup, which comprises of sporangia and spores of the pathogen, can be seen on lower surface of the tainted leaves particularly around the edges of the necrotic injuries. Light to dim chestnut injuries enclose the stems. The influenced stems and petioles get to be powerless at such areas and may fall. Whole harvest gives darkened cursed appearance particularly under illness good conditions and might be demolished inside a week. Tubers in soil get to be tainted by downpour borne sporangia originating from the ailing foliage. Late scourge tainted tubers show unpredictable rosy cocoa to purplish regions which stretch out into interior tissues of the tubers. At the point when a guest goes to a site written in PHP, the server peruses the PHP code and after that procedures it as indicated by its scripted headings [9]. The contaminated tubers more often than not are hard, dry and firm yet may get assaulted by delicate decay bringing about microscopic organisms and decay in field and stores.

Checking populace structure of P. infestans has been on the plan of academic group and new systems have gotten to be accessible. Prior natural markers including mating sorts, race design and metalaxyl affectability were utilized for checking the populace structure of P. infestans. However, now with the presentation of atomic strategies, different markers have picked up significance. The most utilized strategies for late curse populace studies are: Isozymes, RFLP (limitation part length polymorphism), mitochondrial haplotype, AFLP (opened up section length polymorphism) and, SSRs (basic arrangement rehashes, microsatellites).

Leaf spots are round imperfections found on the leaves of numerous types of plants, for the most part brought on by parasitic organisms or microbes. A run of the mill spot is "zonal", which means it has an unmistakable edge and regularly has a darker outskirt. At the point when heaps of spots are available, they can become together and turned into a curse or a smudge. At that point the grape leaf infection division is performed utilizing adjusted self-arranging highlight map with hereditary calculations for streamlining and bolster vector machines for grouping [10]. Contagious spots are as a rule round or freestyle in shape.In most cases, leaf spots are thought to be just a corrective issue, yet they can effectsly affect nurseries or in horticulture. This is found in chestnut takes off.

\section{LITERATURE REVIEWS}

Biswas et.al in [1] those suggested system takes photos of a total about potato relinquishes with complex establishment Similarly as data which are gotten under uncontrolled environment. In this prescribed approach

decorrelation stretching out might be utilized to enhance those shade contrasts in the enter pictures. At that point feathery C-mean gathering is associated will area those ailment affected region which in like manner fuse establishment for same shade qualities.

Adnan et.al in [2] paper presented A database structure of the versatile tree leaf beet diseases which collected under An individual landing page (PHP) script and using sorted out request tongue (MySQL). In this work, there would three picked diseases that camwood an opportunity to be recognized using this database system which are Corynespora leaf beet Spot, lifted spot Furthermore Collectrotichum leaf fat. At first, those information for range about interest (ROI) from each for these three sorts for ailments subordinate upon Red, Green Also blue (RGB) shading model beginning with past examinations will make taken as the focal point of the database structure.

Meunkaewjinda et.al in [3] the proposed structure involves from asserting three major parts: (i) grape leaf beet shade division, (ii) grape leaf fat division, Furthermore (iii) examination and request of afflictions. The grape leaf beet shading division will be pre-handling module which sections out At whatever irrelevant establishment information. A 
self-sorting out trademark delineate for a back-engendering neural framework might be sent on recognize shades about grape leaf beet. This greater part of the information is utilized to section grape leaf beet pixels inside the photo.

Pathak et.al in [4] paper proposed a mixing for neighborhood What's more overall framework to unpredictability picture redesign. Overall many-sided quality picture update upgrades low contrast about picture for an overall methodology. This kind for overall redesign refrains from commotion Also distinctive ringing relics of a propelled picture. For overall intricacy picture redesign The moment that auxiliary difference happens it reasons under acquaintance with deference with a few and just picture and through presentation ahead some other and just a photo. Overall distinction keeping picture redesign need an extraordinary arrangement inclination yet all the it need over adjacent update about picture infers it neglects to offer those area purpose of enthusiasm from guaranteeing a photo. The moment that we utilize adjacent purpose of enthusiasm for a picture, the area purpose of enthusiasm around a photo camwood make described Previously, better best approach. Neighborhood contrast picture redesign increases hullabaloo of a photo when auxiliary intricacy increment happens.

Dong et.al in [5] those theory of cushioned sets need been utilized to oversee picture update issues to defiled pictures over which the photo edges are questionable and wrong. For the people sorts for pictures, with precisely degree, the considerable update effect could be got using the fleecy sets-based picture redesign procedure instead of those traditional picture overhaul strategies.

Ledig et.al in [6] they familiar Patch-based appraisal about picture division (PEIS), an all system will overview division bore. Their method might be reliant after finding patavium correspondences and the associated patavium relocations, which allow the estimation from guaranteeing division isolation racial slant. They measure both those comprehension of as far as possible and the security of the division shape. They encourage review those division complexity inside patches with weight those dedication of neighborhood division likeness of the overall score. They surveyed PEIS as for both fabricated data Also two helpful imaging datasets.

Huang et.al in [7] a solitary imperfection picture division calculation can't get the fancied division results for all pictures due to the deformity assorted qualities. A parallel mix division strategy is proposed to coordinate numerous consequences of the distinctive division calculations to get the coveted deformity division map for rapid aluminum surface imperfection recognition. Two sorts of division calculations are planned in this blend structure, to be specific, the programmed limit division in view of the picture measurable model and versatile entropy-based division.

Biswas et.al in [8] the proposed framework takes pictures of a gathering of potato leaves with complex foundation as information which are caught under uncontrolled environment. In this proposed approach decorrelation extending is

utilized to improve the shading contrasts in the information pictures. At that point Fuzzy C-mean grouping is connected to fragment the malady influenced range which likewise incorporate foundation with same shading qualities. At last we propose to utilize the neural system based way to deal with arrange the ailment influenced locales from the comparable shading textured foundation. The proposed calculation accomplishes a precision of $93 \%$ for 27 pictures caught in various light condition, from various separations and at various introductions alongside complex foundation.

Adnan et.al in [9] paper introduced a database arrangement of the elastic tree leaf maladies which assembled into a Personal Home Page (PHP) script and utilizing Structured Query Language (MySQL). In this work, there are three chosen infections that can be recognized utilizing this database framework which are Corynespora Leaf Spot, Bird's Eye Spot and Collect rotichum Leaf Disease. At first, the data of Region of Interest (ROI) from each of these three sorts of maladies in light of Red, Green and Blue (RGB) shading model from past investigations will be taken as the contribution to the database framework. By utilizing PHP, a server-side dialect which sends Web pages to asking for guests (you, the customer, with your Web program). At the point when a guest goes to a site written in PHP, the server peruses the PHP code and after that procedures it as per its scripted headings. The customer additionally ready to fill in the information through the framework online and the database framework yield will distinguish the sicknesses taking into account client information. The motivation behind advancement of this framework is to help new clients to distinguish the sorts of elastic tree leaf illnesses whereby it is comprises of information accumulation, put away and kept composed by the Database Management System. In this manner, by utilizing the database framework, this may help the customer on distinguishing the sicknesses by contrasting their records and the accessible data. While the benefit of this framework is it might help new clients to examine the qualities of the maladies without alluding to the ability since the data is comprises of test of pictures and its indications.

Meunkaewjinda et.al in [10] the proposed framework comprises of three fundamental parts: (i) grape leaf shading division, (ii) grape leaf ailment division, and (iii) investigation and characterization of maladies. The grape leaf shading division is pre-handling module which fragments out any unimportant foundation data. A self-sorting out component outline with a back-engendering neural system is sent to perceive shades of grape leaf. This data is 
utilized to fragment grape leaf pixels inside the picture. At that point the grape leaf infection division is performed utilizing adjusted self-arranging highlight map with hereditary calculations for enhancement and bolster vector machines for arrangement. At last, the subsequent fragmented picture is separated by Gabor wavelet which permits the framework to break down leaf malady shading highlights more effective. The bolster vector machines are of course connected to order sorts of grape leaf sicknesses. The framework can have the capacity to classify the picture of grape leaf into three classes: scab illness, rust ailment and no ailment. The proposed framework demonstrates alluring results which can be further created for any horticultural item examination/review framework.

Pathak et.al in [11] paper recommended a blend of neighborhood and worldwide technique for complexity picture improvement. Worldwide difference picture upgrade enhances low differentiation of picture universally. This kind of worldwide upgrade maintains a strategic distance from clamor and other ringing relics of an advanced picture. In worldwide differentiation picture improvement when high complexity happens it causes under presentation on some a player in picture and over introduction on some other part of a picture. Worldwide differentiation picture improvement has much favorable position however it need in neighborhood upgrade of picture means it does not have the nearby detail of a picture. When we utilize neighborhood point of interest of a picture, the nearby detail of a picture can be characterized in better way. Nearby difference picture improvement builds commotion of a picture when high complexity pick up happens. When we utilize worldwide differentiation picture upgrade or neighborhood contrast picture improvement without any assistance it is not advantageous but rather when we utilize blend of nearby and worldwide technique it gives us better results for certain pictures. In this paper we will going to utilize worldwide difference extending strategy for worldwide differentiation picture upgrade. In nearby difference picture improvement strategy we are utilizing unsharp veiling method to upgrade the neighborhood subtle element of a picture. The fundamental point of utilizing this mix of nearby and worldwide strategy is to save the brilliance of a picture when differentiation picture improvement is finished.

Dong et.al in [12] the hypothesis of fluffy sets has been utilized to manage picture upgrade issues for debased pictures in which the picture edges are questionable and off base. For those sorts of pictures, to some degree, the great upgrade impact can be gotten utilizing the fluffy sets-based picture improvement strategy rather than the customary picture upgrade approaches. The dim level most extreme has not been changed in the traditional fluffy upgrade strategy proposed by S. K. Buddy, so this strategy is not fit for the improvement issue of debased pictures with less dark levels and low differentiations; the way that the scope of enrollment capacity of dim levels is not standardization structure, i.e. $[0,1]$, is another disservice of the customary fluffy upgrade approach. To manage the issues specified over, a summed up iterative fluffy improvement calculation is proposed in this paper. Another picture quality appraisal model is recommended on the premise of the measurable components of the dim level histogram of pictures to control the iterative methodology of the proposed picture upgrade calculation. PC reenactment results demonstrated this new upgrade strategy is more reasonable than fluffy improvement and dark level change for taking care of the improvement issues of pictures with less dim levels and low complexities.

Ledig et.al in [13] they presented Patch-based Evaluation of Image Segmentation (PEIS), a general technique to survey division quality. Their strategy depends on discovering patch correspondences and the related patch relocations, which permit the estimation of division inclination. They measure both the assention of the division limit and the protection of the division shape. They advance survey the division many-sided quality inside patches to weight the commitment of neighborhood division comparability to the worldwide score. They assessed PEIS on both manufactured information and two restorative imaging datasets. On manufactured divisions of various shapes, they gave proof that PEIS, in contrast with the Dice score, creates more tantamount scores, has expanded affectability and appraisals division inclination precisely. On heart attractive reverberation (MR) pictures, they exhibited that PEIS can assess the execution of a division technique free of the size or unpredictability of the division under thought. On cerebrum MR pictures, they looked at five changed programmed hippocampus division methods utilizing PEIS. At long last, they imagined the division inclination on a choice of the cases.

Huang et.al in [14] a solitary deformity picture division calculation can't acquire the craved division results for all pictures on account of the imperfection differing qualities. A parallel blend division technique is proposed to coordinate numerous consequences of the diverse division calculations to get the sought deformity division map for fast aluminum surface imperfection identification. Two sorts of division calculations are planned in this blend structure, in particular, the programmed edge division in view of the picture factual model and versatile entropy-based division. The programmed limit division calculation distinguishes abandons quickly utilizing the edge parameters acquired by displaying the picture adequately, and the versatile entropy-based division calculation viably identifies deformities utilizing ID data entropy. These two sorts of division calculations keep running in parallel, and their division results are intertwined by an "and" operation. In this way, an enhanced picture division map with higher exactness is gotten. Numerous test results and field applications demonstrate that the parallel mix division calculation is a steady and productive division calculation, which enhances the precision of the first division calculation that it contains. 


\section{IMAGE CONTRAST ENHANCEMENT METHOD}

This strategy for worldwide and nearby differentiation upgrade is proposed which expand the multifaceted nature of the framework. In this examination non sharp concealing must be utilized to get great results which are a superfluous necessity in the framework. In this examination we need to utilize separate neighborhood contrast upgrade to get great results without which they are unachievable. In this exploration additionally the different utilization of worldwide upgrade is required to lessen the commotion as a result of the over reliance of the framework on composition highlights. We can utilize the slope highlight which will lessen the need of utilizing separate worldwide and neighborhood contrast improvements. The issue of need of partitioned non sharp veiling can be tackled by the utilization of slope elements. The issue of isolated utilization of neighborhood and worldwide difference upgrades can be determined by utilizing exceedingly separated inclination highlights. We can utilize the slope highlight to likewise tackle the issue of many-sided quality of the proposed framework.

\section{Parallel Combination Segmentation Method}

This research proposes a segmentation method is proposed which uses two different methods for segmentation. One is Automatic thresholding and the other is Entropy based method. Separate defect detection has to be done by Automatic thresholding to get good results, increasing the complexity of the system. The complexity also increases by the separate use of entropy based method which detects the defects using entropy. We can use the gradient feature which will reduce the need of using separate automatic thresholding and entropy methods. The problem of need of separate defect detection using automatic thresholding can be solved by the use of gradient features. The problem of separate use of defect detection using entropy method can be resolved by using highly differentiated gradient features. We can use the gradient feature to also solve the problem of complexity of the proposed system even in this research.

\section{PROBLEM FORMULATION}

India is an agrarian nation wherein the majority of the populace relies on upon agribusiness. Research in farming is pointed towards increment of profitability and nourishment quality at decreased consumption, with expanded benefit. Rural generation framework is a result of a mind boggling association of soil, seed, and agro chemicals. Vegetables and natural products are the most critical horticultural items. Keeping in mind the end goal to get more profitable items, an item quality control is fundamentally obligatory. Numerous studies demonstrate that nature of agrarian items might be decreased because of plant maladies. Infections are hindrance to the typical condition of the plant that alters or interferes with its essential capacities, for example, photosynthesis, transpiration, fertilization, preparation, germination and so forth. These ailments are brought about by pathogens viz., organisms, microorganisms and infections, and because of unfriendly ecological conditions. After division the region of interest i.e. sick part removed. In the following step, critical components are extricated and those elements can be utilized to decide the importance of a given specimen. Really, picture highlights for the most part incorporates shading, shape and surface elements. As of now a large portion of the analysts focusing on plant leaf surface as the most essential component in ordering plants. A lot of focus is given to classifier and increasing the features. In fact the variety in features is also important to make system efficient. Different types of features need to be tried for better results. Addition of feature according to the extraction method can enhance the overall performance.

Late Blight is the most decimating malady for Potato in the greater part of the potato developing areas on the planet. For ideal utilization of pesticide and to minimize the yield misfortune, the recognizable proof of ailment seriousness is vital. The key commitment here is a calculation to decide the seriousness of Potato Late Blight illness utilizing picture preparing procedures and neural system. The proposed framework takes pictures of a gathering of potato leaves with complex foundation as data which are caught under uncontrolled environment. In this proposed approach decorrelation extending is utilized to enhance the shading contrasts as a part of the information pictures. At that point Fuzzy C-mean bunching is connected to portion the sickness influenced region which additionally incorporate foundation with same shading attributes. At last we propose to utilize the neural system based way to deal with group the malady influenced locales from the comparable shading textured foundation. We propose the use of gradient features along with texture features for the detection of potato late blight. Late blight detection with the help of Image processing is a very covert task which needs very good variation in the feature sets before using it for the final classification.

\section{PROPOSED WORK}


In the present work, we have developed a pattern detection technique using Gradient and Texture Features.

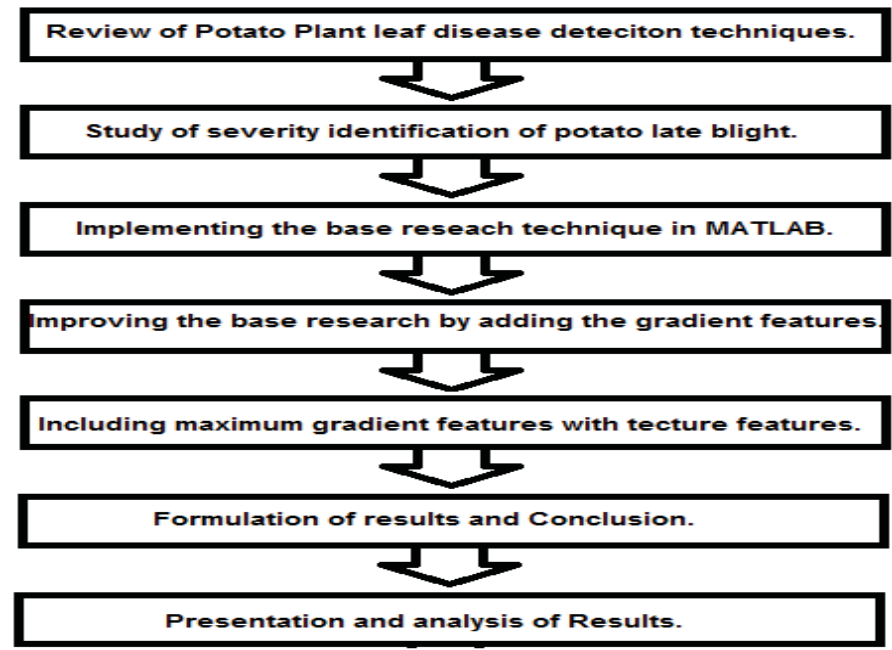

Figure 1: Proposed Methodology

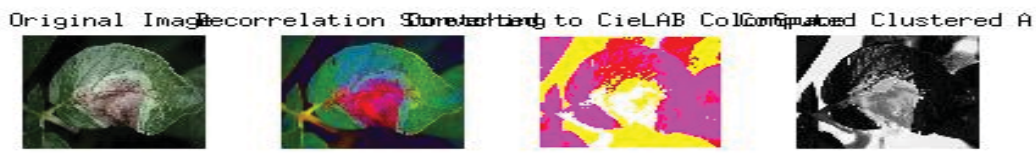

Computed Clustereddmputed Clustered inal Clusted in Mebverted to HSV Space
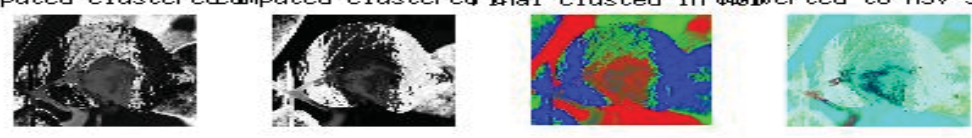

Figure 2: Intermediate Results of Processing the Images

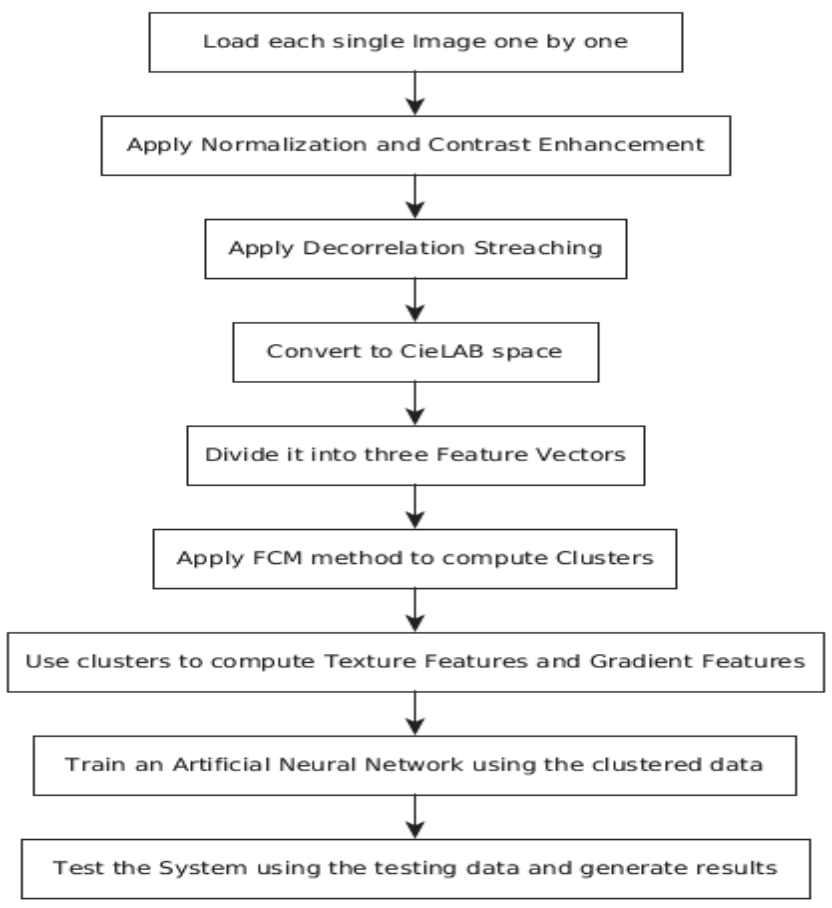


In our method the flowchart of the concept will explain the overall work:

Figure 2: Flowchart of the System.

In the flowchart all the steps are explained as follow:

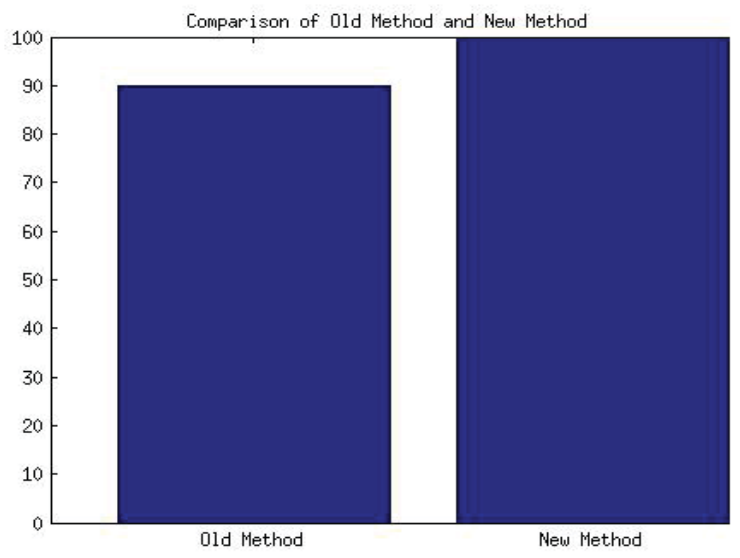

Figure 3: Results are coming out to be $100 \%$ in our case.

We stack every single picture utilizing a circle. We have processed standardized picture and improve the difference. We have connected decor relation extending. We have then changed over it into LAB space. We have then utilized it to characterize an element vector.

We have utilized this information to group utilizing FCM. We have changed over the groups to a HSV picture. We have removed the angle and composition components and use $t$ to prepare ANN.

\section{CONCLUSTION}

In this straight on examination of strategies for plant leave sickness location. We have examined two techniques parallel mix division strategy and Image contrast improvement technique. In the main technique the nearby and worldwide limits are being utilized. The nearby and worldwide complexity upgrade is critical to decrease clamor in the picture and identify the right ROI which is imperative. In the other strategy again two separate methods are connected i.e. the Automatic thresholding and Entropy based strategy. This expands the multifaceted nature of the framework, which was likewise an issue in the primary system. Considering everything the reliance of both the strategy on surface elements is clear yet at the same time offering significance to multifaceted nature we would infer that the principal technique is better one for plant leaf infection discovery. In this paper, we have built up another technique for characterization recently scourge ailment in potato leafs. We have connected inclination and composition highlights keeping in mind the end goal to create $100 \%$ precision..

\section{REFERENCES}

[1] Biswas, S., Jagyasi, B., Singh, B.P. and Lal, M., Severity identification of Potato Late Blight disease from crop images captured under uncontrolled environment. In Humanitarian Technology Conference-(IHTC), IEEE 2014 Canada International (pp. 1-5). IEEE, June, 2014.

[2] Adnan, S.F.S., Abdullah, N.E., Hashim, H., Yusof, Y.W.M. and Malim, M.Y.S., A development of online database system for rubber tree leaf diseases. In Computer Applications and Industrial Electronics (ISCAIE), Symposium on (pp. 312-317). IEEE,December,2012.

[3] Meunkaewjinda, A., Kumsawat, P., Attakitmongcol, K. and Srikaew.A., Grape leaf disease detection from color imagery using hybrid intelligent system. In Electrical Engineering/Electronics, Computer, Telecommunications and Information Technology. ECTI-CON 2008. 5th International Conference on (Vol. 1, pp. 513-516). IEEE,May,2008.

[4] Pathak, S.S., Dahiwale, P. and Padole, G., A combined effect of local and global method for contrast image enhancement. In Engineering and Technology (ICETECH), IEEE International Conference on (pp. 1-5). IEEE,March,2015.

[5] Dong-liang, P. and An-Ke, X., Degraded image enhancement with applications in robot vision. In Systems, Man and Cybernetics, IEEE International Conference on (Vol. 2, pp. 1837-1842). IEEE, October,2015.

[6] Ledig, C., Shi, W., Bai, W. and Rueckert, D., Patch-based evaluation of image segmentation. In Proceedings of the IEEE Conference on Computer Vision and Pattern Recognition (pp. 3065-3072),2014. 
[7] Huang, X.Q., Luo, X.B. and Wang, R.Z., A real-time parallel combination segmentation method for aluminum surface defect images. In Machine Learning and Cybernetics (ICMLC), International Conference on (Vol. 2, pp. 544-549). IEEE,July, 2015.

[8] Dziuk, M.A. and Jamshidi, M., Fuzzy logic controlled UAV autopilot using C-Mean clustering. In System of Systems Engineering (SoSE), 6th International Conference on (pp. 305-310). IEEE,June,2011.

[9] Yang, Y., Wang, R. and Li, J., Research on IT resources fuzzy C-mean clustering based on Resource Based View. In Fuzzy Systems and Knowledge Discovery (FSKD), Seventh International Conference on (Vol. 6, pp. 2895-2899). IEEE,August,2010.

[10] Kondo, T., Ueno, J. and Takao, S., Hybrid feedback GMDH-type neural network using principal component-regression analysis and its application to medical image recognition of heart regions. In Soft Computing and Intelligent Systems (SCIS), Joint 7th International Conference on and Advanced Intelligent Systems (ISIS), 15th International Symposium on (pp. 1203-1208). IEEE, December,2014. 